\title{
Minimizing Radial-velocity Errors caused by Spectral-type Mismatch in Early-type Stars
}

\author{
Werner Verschueren and Marc David \\ University of Antwerp (RUCA), Astrophysics Research Group, \\ Groenenborgerlaan 171, 2020 Antwerpen, Belgium \\ Myriam Vrancken \\ Royal Observatory of Belgium, Ringlaan 3, 1180 Brussel, Belgium
}

\begin{abstract}
We present the first results of a long-term study to increase the external accuracy of (relative and absolute) radial velocities of earlytype stars below the $1 \mathrm{~km} / \mathrm{s}$ level. The accuracy suffers mainly from important systematic errors caused by intrinsic object-template mismatch in cross-correlation. Based on synthetic spectra, the magnitude of these mismatch errors is shown in different spectral regions and for different rotational velocities around spectral-type mid-A. A line selection and combination scheme is proposed which optimizes the accuracy (systematic errors) in view of the precision (random errors) required.
\end{abstract}

\section{Background and long-term project}

The internal radial velocity (RV) precision (random errors) for early-type stars, attainable theoretically and in practice, is limited by a low line density, a high rotational velocity and a complicated cross-correlation peak morphology. More importantly, the morphology of line blends varies rapidly with spectral-type for these stars; spectral-type mismatch is thus more likely and, combined with the low line density, more harmful (systematic errors) in cross-correlation of 'similar' spectral types. In addition, atmospheric velocity fields may influence line positions at the $\mathrm{km} / \mathrm{s}$ level or more for the earliest spectral-types. See Verschueren (1995) for an overview of early-type RV problems.

Until now, very few systematic studies have been carried out to investigate the precision and accuracy attainable for RV work of early-type stars. On the other hand, ongoing efforts to set up an early-type RV standard star system (Latham \& Stefanik 1992; Fekel 1999) require the development of techniques to avoid systematic errors that depend on spectral type.

A few years ago, we initiated a long-term project to characterize and improve the RV precision and accuracy for early-type stars. Conclusive results regarding the optimization of the precision can be found in David \& Verschueren (1995) and Verschueren \& David (1998). This contribution presents the first results of an empirical study to quantify and minimize systematic RV shifts due to object-template mismatch ('mismatch shifts'), which in many cases is the main source of systematic errors. 


\section{Synthetic spectra and cross-correlations}

In a first stage, we use synthetic spectra to quantify and minimize mismatch shifts: they provide an arbitrarily dense grid in $T_{\text {eff }}$ and $\log g$, they cover a large wavelength range, their RV is exactly zero and they are noise-free. Although real spectra differ from the models, we expect differential effects to be realistic to first order. And although real spectra may contain additional mismatch due to physical processes not included in the models (winds, abundance peculiarities, magnetic fields, velocity fields, ...), it is instructive to separate the temperature/gravity mismatch from other factors and investigate the latter on real spectra.

Kurucz LTE line-blanketed atmospheres of solar composition are used (Kurucz 1993) to produce LTE synthetic spectra with the programme SYNSPEC (Hubeny et al. 1994) in the wavelength range $3700-5200 \AA$. The resolution is $28 \times 10^{-6}$ in $\ln (\lambda)( \pm 0.12 \AA / p$ at $4300 \AA)$. Cross-correlations are done with the CORSPEC package, updated from Verschueren (1991).

\section{One example: attainable accuracy at mid-A}

\subsection{Definition of spectral-type mismatch}

As template, we take a synthetic spectrum of fixed $T_{\text {eff }}=8500 \mathrm{~K}$ and $\log g=$ 4.0 (corresponding roughly to A5V). Fourteen different object spectra are made by assuming an expected uncertainty of the difference in $T_{\text {eff }}$ of $500 \mathrm{~K}$ (we took $\left.T_{\text {eff }}=8000,8250,8500,8750,9000 \mathrm{~K}\right)$ and in $\log g$ of $0.2 \operatorname{dex}(\log g=3.8,4.0,4.2)$; this roughly corresponds to A7 - A3 and IV - ZAMS. In addition, we consider 6 different rotational velocities $(V \sin i=5,50,100,150,200,300 \mathrm{~km} / \mathrm{s})$, assuming no rotational mismatch between object and template.

\subsection{Selection of spectral regions}

Continuum regions are defined as those spectral intervals where the normalized flux is above 0.97 over a minimum span of 10 pixels. This restriction is important to avoid end-mismatch effects. Individual spectral regions are then defined as regions in between continuum regions, and chosen with the constraint that they are reasonably long (typically $35 \AA$ for regions without $\mathrm{H}$ lines) and that they are defined as consistently as possible for different $V \sin i$.

\subsection{Cross-correlations}

The 14 object spectra are correlated with the template in each of the chosen spectral regions, and independently for each $V \sin i$. For each region then and for each $V \sin i$, we define the expected systematic error $E_{\mathrm{RV}}$ as the maximum of the mismatch shifts over all 14 'types' of mismatch. A number of conclusions can be drawn: shifts are extremely $V \sin i$ dependent; for $V \sin i>100 \mathrm{~km} / \mathrm{s}$, most $E_{\mathrm{RV}}>1 \mathrm{~km} / \mathrm{s}$, but even up to $V \sin i=200 \mathrm{~km} / \mathrm{s}$ there are some (very few) $E_{\mathrm{RV}}<0.5 \mathrm{~km} / \mathrm{s} ;$ for $V \sin i>100 \mathrm{~km} / \mathrm{s}$, most random errors exceed $1 \mathrm{~km} / \mathrm{s}$ (for $(S / N)_{\text {obj }}=100$ and $(S / N)_{\text {tem }}=\infty$ ); regions with $\mathrm{H}$ lines have larger shifts on average due to $\log g$ mismatch, which counters their advantage of causing smaller random errors; for almost all regions, the shift varies monotonically 

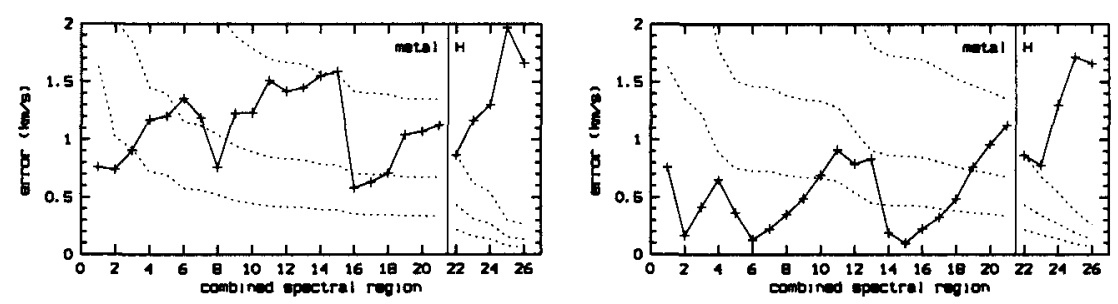

Figure 1. a. Maximum mismatch shifts $\left(E_{\mathrm{RV}}\right)$ for all 'naturally' combined spectral regions at $V \sin i=150 \mathrm{~km} / \mathrm{s}$. Results are shown separately for combinations without and with $\mathrm{H}$ lines. Random errors are given (dashed line) for a $(S / N)_{\mathrm{obj}}=50,100,200$ from top to bottom respectively, and a $(S / N)_{\text {tem }}=\infty$. b. Idem for the 'clever' combinations

with $\Delta T_{\text {eff }}$ and with $\Delta \log g$; if $V \sin i>100 \mathrm{~km} / \mathrm{s}$, the sign of the mismatch shift for a given $\Delta T_{\text {eff }}, \Delta \log g$ is the same in most of the regions, presumably due to an asymmetry in the line blends induced by positional relations between the contributing multiplets in different regions.

\subsection{Combining spectral regions}

The most 'natural' set of combined regions (for each $V \sin i$ ) is constructed by defining the first combined region as the individual region with the smallest $E_{\mathrm{RV}}$, while subsequent combined regions are made by adding one by one the individual regions ordered according to their $E_{\mathrm{RV}}$. The last combined region is thus the complete spectrum. Figure la shows $E_{\mathrm{RV}}$ for each combined region. The conclusion is that, although random errors may be reduced considerably, individual regions do not cancel out but can easily conspire to produce higher systematic shifts; and omitting the regions with the highest individual shifts does not guarantee a lower total shift.

A more 'clever' set of combined regions can be constructed by adding one after the other the individual region which minimizes $E_{\mathrm{RV}}$ for the resulting combined region (Figure $1 \mathrm{~b}$ ). This results in significantly smaller mismatch shifts, although the performance of these combined regions must be verified on real spectra (see Section 2).

\subsection{Attainable accuracy as a function of $V \sin i$}

The set of 'cleverly' combined regions was then applied to intermediate $V \sin i$ values to check their robustness and thus validity (e.g. the performance of the $V \sin i=100 \mathrm{~km} / \mathrm{s}$ combined regions was checked at $V \sin i=75$ and $125 \mathrm{~km} / \mathrm{s}$ ). We conclude that the 'cleverly' combined regions work quite well also at different $V \sin i ; E_{\mathrm{RV}}$ values are only slightly higher than expected from the $E_{\mathrm{RV}}$ values at the original $V \sin i$ gridpoints. Moreover, within each $V \sin i$ range $(5-30,30-$ $75,75-125,125-175,175-250,250-300)$, there exists a number of common combined regions that produce among the lowest shifts for all $V \sin i$ values in that range. Figure 2 shows these optimal attainable accuracies as measured on the intermediate $V \sin i$ values, i.e. in the worst case. 


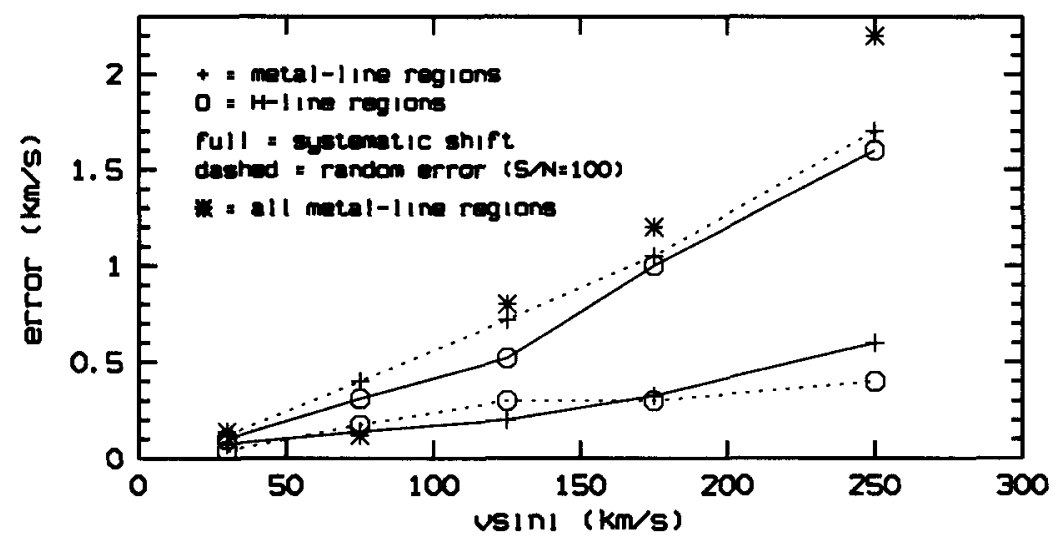

Figure 2. Minimal attainable systematic mismatch error and accompanying random error $\left((S / N)_{\mathrm{obj}}=100\right.$ and $\left.(S / N)_{\mathrm{tem}}=\infty\right)$ as a function of $V \sin i$. Results from combining spectral regions with and without $\mathrm{H}$ lines are shown separately. Also shown is the systematic shift from a combination of all metal line regions together: it leads to much higher errors, except at $V \sin i=75 \mathrm{~km} / \mathrm{s}$ where strong accidental canceling occurs.

This work started from a purely technical point of view; its results should now be reconsidered in the light of our physical knowledge of line formation; a better understanding of why certain wavelength regions are more suitable than others should allow us to refine and strengthen the present results.

Acknowledgments. We appreciated enlightening discussions with Herman Hensberge and Elizabeth Griffin. Financial support for this work was obtained from the Special Research Fund (BOF/NOI) of the University of Antwerp and from the Fund for Scientific Research (FWO) - Flanders (Belgium). W.V. is a Postdoctoral Fellow of the latter Fund.

\section{Discussion}

Dravins: Ordinarily one correlates the template with the object spectrum in the sense of the presence of photons in that spectrum. One may also correlate the template with the 'negative' of the object spectrum, i.e. its absorbed light measured down from the continuum. For asymmetric spectral features, the wavelength shift will have different signs in these two cases. Perhaps a combination of 'positive' and 'negative' correlations could be used to improve the stability against spectral-type mismatch?

Verschueren: I have never looked into that, although my first idea would be that you simply get the same shift but with opposite sign, which would not be very useful.

Latham: We have considerable experience with trying to match observed spectra to synthetic calculated templates. For rich data sets we find that we can iden- 
tify the optimum effective temperature (and/or metallicity and/or gravity) to a precision better than the spacing of our grid. Of course, there is a degeneracy between, for example, effective temperature and metallicity, but we hope that the effects of template mismatch can be limited to the spacing of our grid for the well-observed cases.

Verschueren: Allowing for a somewhat larger mismatch is necessary to be able to correlate two observed stars with each other of 'the same' or 'similar' spectral type. Also, considering the existence of other types of mismatch in observed spectra, such as abundance differences and other spectral peculiarities, it seems appropriate not to narrow the amount of mismatch too much in the synthetic spectra.

\section{References}

David, M. \& Verschueren, W. 1995, A\&A Suppl. 111, 183

Fekel, F. 1998, this meeting

Hubeny, I., Lanz, T. \& Jefferey, C.S. 1994, Newsl. Anal. Astron. Sp. 20, 30

Kurucz, R.L. 1993, S.A.O. CD-ROM 13

Latham, D.W. \& Stefanik, R.P. 1992, Trans. IAU, Vol. XXIB, 269

Verschueren, W. 1991, Ph.D. Thesis, Free University of Brussels (VUB), Belgium

Verschueren, W. 1995, Standard Star Newsletter 18, 6

Verschueren, W.\& David, M. 1998, A\&A, submitted 